\title{
Cluster cross sections from pickup measurements: Are the established methods consistent?
}

\author{
J. Fedor, ${ }^{\text {a) }}$ V. Poterya, A. Pysanenko, and M. Fárník ${ }^{\mathrm{b}}$ \\ J. Heyrovský Institute of Physical Chemistry v.v.i., Academy of Sciences of the Czech Republic, Dolejškova 3, \\ 18223 Prague, Czech Republic
}

\begin{abstract}
Pickup of several molecules, $\mathrm{H}_{2} \mathrm{O}, \mathrm{HBr}$, and $\mathrm{CH}_{3} \mathrm{OH}$, and $\mathrm{Ar}$ atoms on free $\mathrm{Ar}_{N}$ clusters has been investigated in a molecular beam experiment. The pickup cross sections of the clusters with known mean sizes, $\bar{N} \approx 150$ and 260 were measured by two independent methods: (i) the cluster beam velocity decrease due to the momentum transfer of the picked up molecules to the clusters, and (ii) Poisson distribution of a selected cluster fragment ion as a function of the pickup pressure. In addition, the pickup cross sections were calculated using molecular dynamics and Monte Carlo simulations. The simulations support the results of the velocity measurements. On the other hand, the Poisson distributions yield significantly smaller cross sections, inconsistent with the known $\operatorname{Ar}_{N}$ cluster sizes. These results are discussed in terms of: (i) an incomplete coagulation of guest molecules on the argon clusters when two or more molecules are picked up; and (ii) the fragmentation pattern of the embedded molecules and their clusters upon ionization on the Ar cluster. We conclude that the Poisson distribution method has to be cautiously examined, if conclusions should be drawn about the cluster cross section, or the mean cluster size $\bar{N}$, and the number of picked up molecules.
\end{abstract}

\section{INTRODUCTION}

The pickup technique was introduced in mid 1980s for doping clusters with foreign molecules. ${ }^{1}$ Since then it has been developed into a very powerful tool in the cluster science: especially numerous experiments with large helium clusters use this technique ${ }^{2-4}$ to study the spectroscopy of molecules ${ }^{5-10}$ and their reactions ${ }^{11-15}$ in the cold superfluid environment of the helium nanodroplets. Here we focus on the pickup to argon clusters which was also exploited in many studies, e.g., for cluster isolated chemical reactions on large Ar clusters in the group of Mestdagh ${ }^{16,17}$ and others. ${ }^{18}$ The pickup to argon and various other clusters was also employed for studies of photochemistry of molecules in cluster environment, ${ }^{19-22}$ and in general for investigation of various mixed molecular clusters. ${ }^{23,24}$ Despite these widespread applications of the pickup technique and the earlier studies of this process, ${ }^{2-4}$ many questions remain opened concerning the mechanism of the pickup process itself, and sticking, migration and coagulation of the molecules on/in the host clusters - especially for other cluster species than helium nanodroplets. This paper contributes to the detailed understanding of this technique by investigating the pickup process of various molecules $\left(\mathrm{HBr}, \mathrm{H}_{2} \mathrm{O}, \mathrm{CH}_{3} \mathrm{OH}\right)$ and argon atoms on large argon clusters.

The second crucial aspect of our paper concerns the pickup cross section and the corresponding cluster size de-

\footnotetext{
a) Author to whom correspondence should be addressed. Electronic mail: juraj.fedor@unifr.ch. Present address: Department of Chemistry, University of Fribourg, Chemin du Musée 9, CH-1700 Fribourg, Switzerland.

b) Author to whom correspondence should be addressed. Electronic mail: michal.farnik@jh-inst.cas.cz.
}

termination. One of the original motivations for cluster studies has been to investigate the evolution of certain properties or processes as a function of the system size. ${ }^{25}$ Therefore the cluster size determination has been one of the pivotal issues in the cluster science, which is not a straightforward task for the neutral clusters. Generally, due to the strong fragmentation upon ionization of the weakly bound species, mass spectrometry is of little help here. ${ }^{26-28}$ For the small clusters $N \lesssim 10$ the scattering method of Buck and Meyer ${ }^{26,27}$ can be exploited to measure the neutral cluster sizes. For the large and medium sizes $\left(N \sim 10-10^{3}\right)$ several other methods have been developed, e.g., electron diffraction, ${ }^{29-32}$ cluster beam scattering by a buffer gas, ${ }^{33}$ pickup of sodium atoms and photoionization, ${ }^{34}$ and helium atom diffraction on clusters. ${ }^{35}$

Here we are concerned with two methods for the mean cluster size determination using the pickup process: (1) The first method consists in measuring the variations of the average beam velocity with the pickup pressure. ${ }^{36}$ (2) The second one relies on measuring the Poisson distributions of the picked up molecules. ${ }^{37}$ Their common feature is that in order to evaluate the cluster mean size $\bar{N}$ from the measured data, the pickup cross section $\sigma$ has to be determined independently - generally, the geometrical cross section of a hard-sphere was assumed. We assess the two methods by inverting the procedure, i.e., by preparing cluster beam with known mean size $\bar{N}$, evaluating the pickup cross sections $\sigma$ and comparing them.

We utilized large argon $\operatorname{Ar}_{N}$ clusters with known mean cluster sizes $\bar{N}=150$ and 260. The argon clusters have been selected as cluster archetypes for several reasons. First, their size distributions were studied in great detail by various methods ${ }^{35,38-40}$ and well characterized by Hagena's scaling law. ${ }^{35,39}$ Therefore the cluster sizes determined by the 
expansion conditions are well known. Further advantage of $\operatorname{Ar}_{N}$ clusters is, that a fair amount of work exists on the pickup of the present molecules in particular: $\mathrm{HBr},{ }^{41} \mathrm{H}_{2} \mathrm{O},{ }^{37}$ $\mathrm{CH}_{3} \mathrm{OH} .{ }^{18}$ This facilitates a critical comparison of the present data obtained by two different methods. Finally, the interaction of Ar-clusters with pickup molecules and atoms can be described by relatively simple potentials and the pickup can be modelled by molecular dynamics and Monte Carlo methods. The theoretically calculated cross sections provide useful references for the assessment of the two experimental methods. Surprisingly the two experimental methods delivered different results. We conclude that the results from velocity measurements are close to the real cross sections while the Poisson distribution method is biased by an incomplete coagulation of the host molecules on the cluster and fragmentation pattern of the host embedded clusters upon the ionization. Also a combination of the two methods is proposed which, in principle, would allow to determine $\bar{N}$ and $\sigma$ from the experimental data independently without any assumptions of their analytical relation.

\section{EXPERIMENT}

The principle of the experiment can be briefly summarized as follows: clusters are doped with the guest molecules in a pickup chamber and two characteristics of the cluster beam are measured as a function of the pickup gas pressure the neutral beam velocity and the fragment ion mass spectra. Our complex cluster beam apparatus was described in detail elsewhere, ${ }^{42,43}$ therefore only the parts of the machine relevant for the present experiment are described here and shown in Fig. 1.

The argon clusters were generated in the source chamber S1 by supersonic expansion through a conical nozzle (diameter $d=50 \mu \mathrm{m}$, length $2 \mathrm{~mm}$, and opening angle $\alpha=30^{\circ}$ ). The nozzle was cooled to a constant temperature of $T_{0}=223 \mathrm{~K}$ and the size of the clusters was controlled by varying the stagnation pressure of argon $p_{0}$. The resulting mean cluster size can be determined according to the empirical formulas, ${ }^{35,39}$

$$
\bar{N}=K\left(\frac{\Gamma^{*}}{1000}\right)^{\zeta}, \quad \Gamma^{*}=\frac{p_{0}[\mathrm{mbar}] d_{e}[\mu \mathrm{m}]^{0.85}}{T_{0}[\mathrm{~K}]^{2.2875}} K_{c}
$$

where $K=38.4$ and $\zeta=1.64$ were determined from the diffractive $\mathrm{He}$ atom scattering on large $\mathrm{Ar}_{N}$ clusters, ${ }^{35}$ $K_{c}=1646$ is a characteristic constant of the expanding gas for $\operatorname{Ar}$, and $d_{e}=\frac{d}{\tan (\alpha / 2)}$ is the equivalent nozzle diameter. The applicability of these formulas for rare gas clusters were proved by several experiments, ${ }^{35,38-40}$ some of which were done in Göttingen ${ }^{35}$ using the cluster source implemented in the present experiments. The two values of stagnation pressure applied in the present experiments $p_{0}=5$ and 7 bars correspond to the mean cluster sizes $\bar{N}=150$ and 260, respectively.

The Ar cluster beam passed through a skimmer with $1 \mathrm{~mm}$ opening before entering the differentially pumped scattering chamber SC. This chamber served as a pickup cell filled with the gas to dope the clusters with molecules. The effective capture length was $L=17 \mathrm{~cm}^{44}$ The pressure in the chamber was monitored by Bayard-Alpert ionization gauge (Varian type 571), which was calibrated for the specific gases by comparing the measured pressures to the values of a capacitance gauge pressure (Pfeiffer CMR 365). The background pressure in the pickup chamber was $\leq 1 \times 10^{-6} \mathrm{mbar}$, and the pressure with the pickup gas went up to $7 \times 10^{-4}$ mbar.

For the velocity measurements the cluster beam was modulated by a pseudorandom mechanical chopper ${ }^{45}$ (PRC) in the next differentially pumped chopper chamber $\mathrm{CC}$. The chopper contained two pseudorandom sequences of 127 elements and its rotation with frequency of $492.1 \mathrm{~Hz}$ corresponded to a single opening time window of $8 \mu \mathrm{s}$. After the chopper the beam passed the flight path of $955 \mathrm{~mm}$ through the photodissociation chamber PDC (not used in the present experiments) to the ion source of a quadrupole mass spectrometer (QMS) with an electron multiplier detector at the end. The arrival time to the detector was measured. The total flight time was properly corrected for the time spent by the ion fragment in the quadrupole and for any electronic delay of the trigger signals and converted to the beam velocity distribution. The velocity distribution was evaluated from the measured data by the cross-correlation mathematical method. ${ }^{45}$ Usually the quadrupole mass spectrometer was set on the major ionization fragment of Ar clusters which is the dimer $\mathrm{Ar}_{2}^{+}$ $(\mathrm{m} / \mathrm{z}=80 \mathrm{amu})$. The maximum velocity of the Ar clusters (without any pickup gas) measured at the nozzle temperature $T_{0}=223 \mathrm{~K}$ was $490 \mathrm{~ms}^{-1}$ with a speed-ratio of $S=35$. This

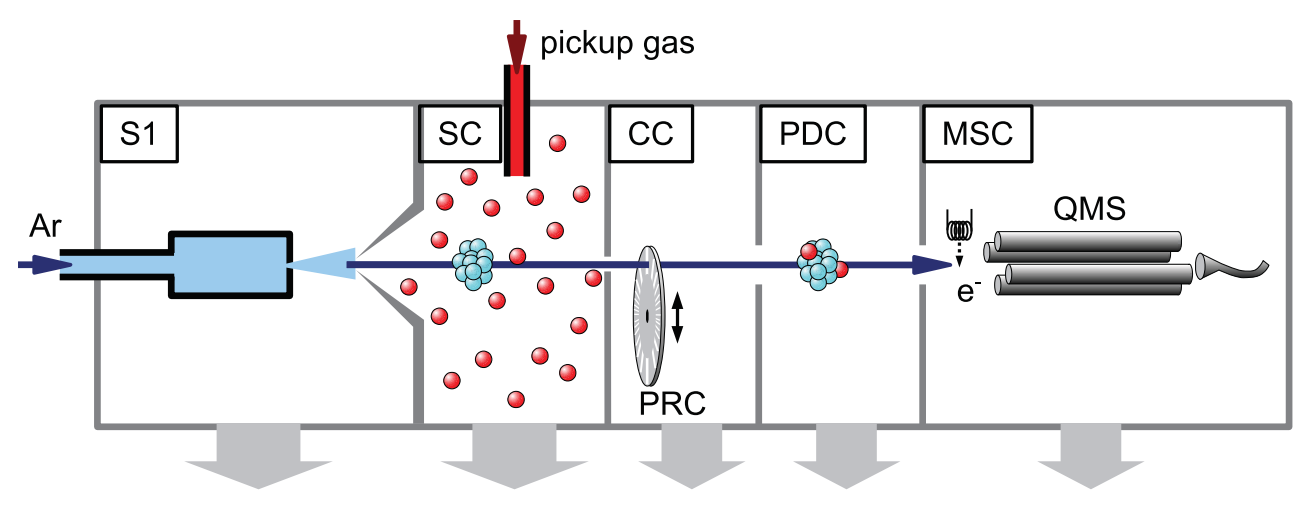

FIG. 1. Schematic picture of the experiment. 
corresponds to the theoretical maximum beam velocity, ${ }^{46}$

$$
v_{\infty}=\sqrt{\frac{2 k_{B} T_{0}}{m} \frac{\gamma}{\gamma-1}}
$$

where $k_{B}$ is the Boltzmann constant and $\gamma$ is the adiabatic index $\left(\gamma=5 / 3\right.$ for a monoatomic ideal gas), i.e., $v_{\infty}$ $=481 \mathrm{~ms}^{-1}$. The slightly higher measured beam velocity can be due to the condensation effect which provides an additional heating of the beam during the expansion.

In addition to the beam velocities, the fragment ion mass spectra of the doped clusters were recorded as a function of the pickup gas pressure. The ionizing electron energy was $70 \mathrm{eV}$. The quadrupole mass spectrometer transmittance was calibrated using the known spectrum of mass calibrant FC-43.

\section{PICKUP DYNAMICS AND STATISTICS}

Since the argon clusters have a solid-like character, ${ }^{29,30,32}$ the molecules do not penetrate the cluster upon the pickup and stay in the outer solvation shell, ${ }^{47-49}$ and the clusters in the beam are slowed down by the pickup due to the momentum transfer. The velocity change increases with the number of guest molecules.

Let us assume an $\operatorname{Ar}_{N}$ cluster of a size $N$ with an initial velocity $v_{i}$ colliding with $k$ stationary molecules in the pickup cell which stick to the cluster. Then the momentum conservation yields for the cluster final velocity $v_{f}$ the equation,

$$
N m_{\mathrm{Ar}} v_{i}=\left(N m_{\mathrm{Ar}}+k m_{X}\right) v_{f},
$$

where $m_{\mathrm{Ar}}$ is Ar atom mass and $m_{X}$ is the mass of the guest molecule. It assumes that no considerable evaporation of cluster constituents occurs upon the pickup. This assumption has been justified by the molecular dynamics simulations outlined in Sec. V. The simulations also show that the molecule remains on the cluster if a considerable momentum transfer between the molecule and the cluster occurs. Thus this simple model describes the final velocity dependence sufficiently well (see Sec. V).

The number of picked up molecules along the path-length $L$ in a gas at a pressure $p$ (corresponding to the number density $n_{g}=p / k_{B} T$ ) can be expressed as

$$
k=n_{g} \sigma_{e} L=\frac{p}{k_{B} T} \sigma_{e} L,
$$

where $\sigma_{e}$ is the pickup cross section. The measured effective cross section $\sigma_{e}$ incorporates the velocity-averaging correction factor ${ }^{46,50,51}$ into the cross section $\sigma_{e}=\sigma_{0} F_{a 0}(\infty, x)$. Combining Eqs. (3) and (4), the relative change in the cluster velocity is directly proportional to pickup cell pressure,

$$
\frac{\Delta V}{V} \equiv \frac{v_{i}-v_{f}}{v_{f}}=\frac{m_{X}}{N m_{A r}} \frac{L \sigma_{e}}{k_{B} T} p .
$$

Thus, from the slope of the relative velocity change dependence on the pressure the pickup cross section $\sigma_{e}$ can be evaluated.

Since the pickup of the $k$ th molecule is assumed to be a random process independent of the previous $(k-1)$ events,

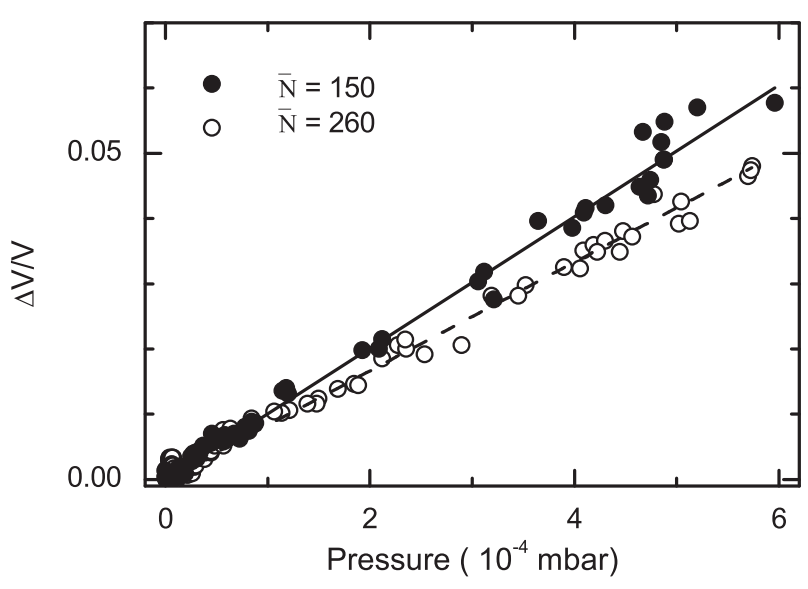

FIG. 2. Relative decrease in the cluster beam velocity as a function of the pickup gas pressure (lines, Eq. (5)) for the pickup of $\mathrm{H}_{2} \mathrm{O}$ on $\mathrm{Ar}_{N}$ clusters with $\bar{N}=150$ and 260 .

its probability follows the Poisson distribution: ${ }^{2}$

$$
P_{k}(p)=\frac{1}{k !}\left(\sigma_{e} L n_{g}\right)^{k} e^{-\sigma_{e} L n_{g}}=\frac{1}{k !}\left(\frac{\sigma_{e} L}{k_{B} T} p\right)^{k} e^{-\frac{\sigma_{e} L}{k_{B} T} p} .
$$

Thus the pickup cross section $\sigma_{e}$ can be obtained by measuring the dependence of a fragment ion intensity (corresponding to, e.g., pickup of $k=1$ molecule) on the pickup pressure, and fitting this dependence with the corresponding Poisson distribution. ${ }^{3}$

\section{EXPERIMENTAL RESULTS}

\section{A. Velocity measurements}

Water $\mathrm{H}_{2} \mathrm{O}$, methanol $\mathrm{CH}_{3} \mathrm{OH}$, hydrogen bromide $\mathrm{HBr}$, and argon Ar were used as pickup gases. According to Eq. (5) the relative change in the cluster beam velocity is directly proportional to the pickup gas pressure. This linear dependence is demonstrated in Fig. 2 for the pickup of $\mathrm{H}_{2} \mathrm{O}$ on $\mathrm{Ar}_{N}$, $\bar{N}=150$ and 260. The somewhat larger scatter of the data for $\bar{N}=150$ corresponds to the smaller signals measured for the smaller clusters. Nevertheless, both data sets can be fitted by straight lines with high confidence, in accordance with the previous experiments. ${ }^{36}$ Similar dependence was measured for the other pickup species. The pickup cross sections $\sigma_{e}$ were derived from the slopes of the linear fits and are summarized in Table I. The error in the velocity measurement is smaller than $1 \%$; however, the uncertainty due to the pressure calibration can be up to $10 \%$.

The quantity that is evaluated directly from the experimental data is the effective cross section,

$$
\sigma_{e}=\sigma_{0} \cdot F_{a 0}(\infty, x),
$$

which incorporates the velocity-averaging correction factor $F_{a 0}$ due to the velocity distribution of the target molecules. ${ }^{46,50,51}$ Label $\infty$ denotes the hard sphere potential approximation, and $x=v_{i} / \alpha_{g}$ where $v_{i}$ is the cluster beam velocity and $\alpha_{g}$ is the most probable velocity 
TABLE I. Pickup cross sections for two mean $\operatorname{Ar}_{N}$ cluster sizes $\bar{N}=150$ and 260 obtained by various methods: velocity measurements, Poisson distribution measurements, and molecular dynamics simulations. The experimental error of $\sigma_{e}$ obtained from the velocity measurements was estimated $\lesssim 10 \%$. The Poisson distribution cross section was obtained by fitting the experimental data by $P_{1}(p)$, Eq. (6), and does not have a real physical meaning as argued in the text. The corrected cross sections $\sigma_{0}$ were evaluated from the experimental $\sigma_{e}$ obtained from the velocity measurements divided by the velocity correction factors $F_{a 0}(\infty, x)$ (see the text). Correction $R_{0}$ to the hard sphere geometrical cross section was evaluated from the simulated cross sections.

\begin{tabular}{|c|c|c|c|c|c|c|c|c|}
\hline Molecule & $\bar{N}$ & $\begin{array}{c}\text { Velocity } \\
\sigma_{e} \\
\left(\AA^{2}\right)\end{array}$ & $\begin{array}{c}F_{a 0} \\
(\infty, x)\end{array}$ & $\begin{array}{c}\sigma_{0} \\
\left(\AA^{2}\right)\end{array}$ & $\begin{array}{c}\text { Detected ion } \\
\text { mass } \\
\text { (amu) }\end{array}$ & $\begin{array}{c}\text { Poisson } \\
\sigma_{e} \\
\left(\AA^{2}\right)\end{array}$ & $\begin{array}{c}\text { Simulations } \\
\sigma_{e} \\
\left(\AA^{2}\right)\end{array}$ & $\begin{array}{l}R_{0} \\
(\AA)\end{array}$ \\
\hline \multirow[t]{2}{*}{$\mathrm{HBr}$} & 150 & 672 & \multirow[t]{2}{*}{1.133} & 593 & \multirow[t]{2}{*}{$\mathrm{Br}^{+}(81)$} & 500 & 748 & \multirow[t]{2}{*}{4.3} \\
\hline & 260 & 836 & & 738 & & 352 & $\ldots$ & \\
\hline \multirow[t]{2}{*}{$\mathrm{H}_{2} \mathrm{O}$} & 150 & 848 & \multirow[t]{2}{*}{1.545} & 549 & \multirow[t]{2}{*}{$\mathrm{H}_{2} \mathrm{O}^{+}(18)$} & 528 & 928 & \multirow[t]{2}{*}{6.1} \\
\hline & 260 & 1083 & & 701 & & 408 & $\ldots$ & \\
\hline \multirow[t]{2}{*}{$\mathrm{CH}_{3} \mathrm{OH}$} & 150 & 1041 & \multirow[t]{2}{*}{1.329} & 783 & \multirow[t]{2}{*}{$\mathrm{CH}_{3} \mathrm{O}^{+}(31)$} & 561 & $\ldots$ & \\
\hline & 260 & 1523 & & 1146 & & 522 & $\ldots$ & \\
\hline \multirow[t]{2}{*}{$\mathrm{Ar}$} & 150 & 708 & \multirow[t]{2}{*}{1.267} & 559 & $\ldots$ & $\ldots$ & 824 & 5.1 \\
\hline & 260 & 1080 & & 852 & $\ldots$ & $\ldots$ & $\ldots$ & \\
\hline
\end{tabular}

in the Maxwellian distribution of the scattering gas. These correction factors were tabulated in the literature ${ }^{50,51}$ and are summarized together with the evaluated $\sigma_{0}$ in Table I.

\section{B. Poisson distributions}

Generally the mass spectra of $\operatorname{Ar}_{N}$ clusters doped with the above molecules exhibit three groups of mass peaks: (i) the most pronounced is the sequence of $\mathrm{Ar}_{n}^{+}$peaks; (ii) the ion fragments of the guest molecules and ion fragments of the formed guest clusters; (iii) in some cases there are mixed cluster ions containing Ar-atom(s) and the picked up molecule or its fragment.

When methanol is introduced into the pickup chamber characteristic peaks at $m / z=31$ amu $\left(\mathrm{CH}_{2} \mathrm{OH}^{+}\right)$and $29 \mathrm{amu}\left(\mathrm{COH}^{+}\right)$appear in the mass spectra. At elevated methanol pressures new peaks raise due to the methanol cluster generation on Ar cluster: 33 amu $\left(\left(\mathrm{CH}_{3} \mathrm{OH}\right) \mathrm{H}^{+}\right)$and $63 \mathrm{amu}\left(\mathrm{CH}_{3} \mathrm{OH} \cdot \mathrm{CH}_{2} \mathrm{OH}^{+}\right)$which can be generated from the methanol dimer and larger clusters $\left(\mathrm{CH}_{3} \mathrm{OH}\right)_{\mathrm{k}}, k \geq 2$; $65 \mathrm{amu}\left(\left(\mathrm{CH}_{3} \mathrm{OH}\right)_{2} \mathrm{H}^{+}\right)$and $95 \mathrm{amu}\left(\left(\mathrm{CH}_{3} \mathrm{OH}\right)_{2} \cdot \mathrm{CH}_{2} \mathrm{OH}^{+}\right)$ which indicate the presence of $k \geq 3$ host clusters; and 97 amu $\left(\left(\mathrm{CH}_{3} \mathrm{OH}\right)_{3} \mathrm{H}^{+}\right)$fragmenting from $k \geq 4$. There was no evidence for $\mathrm{Ar}_{n}\left(\mathrm{CH}_{3} \mathrm{OH}\right)_{\mathrm{m}}{ }^{+}$fragments. Similar spectra were measured for methanol pickup on Ar clusters in other experiments. ${ }^{18}$

From the earlier studies ${ }^{52-55}$ of hydrogen bonded clusters, it was found that a fast proton transfer reaction follows the cluster ionization. Thus the major ionization channel results in the protonated fragments with the abstraction of one or more monomer units. The ions below the monomer mass are usually expected to originate from the ionization of a single molecule on the cluster. The intensities of the following ion peaks were plotted against the pressure in the pickup chamber and analysed with the Poisson statistics, see Fig. 3: $\mathrm{COH}^{+}$and $\mathrm{CH}_{2} \mathrm{OH}^{+}$monitoring the pickup of $k=1$ molecule; $\left(\mathrm{CH}_{3} \mathrm{OH}\right) \mathrm{H}^{+}$and $\left(\mathrm{CH}_{3} \mathrm{OH}\right) \mathrm{CH}_{2} \mathrm{OH}^{+}$monitoring $k$ $=2$ pickup, and $\left(\mathrm{CH}_{3} \mathrm{OH}\right)_{2} \mathrm{H}^{+}$for $k=3$. This assumes the coagulation of molecules upon pickup on the cluster and the following fragmentation upon ionization:

$$
\begin{aligned}
& \mathrm{CH}_{3} \mathrm{OH} \rightarrow \mathrm{COH}^{+}, \mathrm{CH}_{2} \mathrm{OH}^{+}, \\
& \left(\mathrm{CH}_{3} \mathrm{OH}\right)_{2} \rightarrow\left(\mathrm{CH}_{3} \mathrm{OH}\right) \mathrm{H}^{+},\left(\mathrm{CH}_{3} \mathrm{OH}\right) \mathrm{CH}_{2} \mathrm{OH}^{+}, \\
& \left(\mathrm{CH}_{3} \mathrm{OH}\right)_{3} \rightarrow\left(\mathrm{CH}_{3} \mathrm{OH}\right)_{2} \mathrm{H}^{+},
\end{aligned}
$$

i.e., no population of these fragments from larger clusters. We argue in the discussion that our data indicate that these assumptions are not fulfilled completely.

The capture of water molecules results in the observation of peaks at $m / z=18 \mathrm{amu}\left(\mathrm{H}_{2} \mathrm{O}^{+}\right)$and $58 \mathrm{amu}\left(\mathrm{Ar} \cdot \mathrm{H}_{2} \mathrm{O}^{+}\right)$ presumably from the monomer; $19 \mathrm{amu}\left(\mathrm{H}_{3} \mathrm{O}^{+}\right)$and $59 \mathrm{amu}$ $\left(\mathrm{Ar} \cdot \mathrm{H}_{3} \mathrm{O}^{+}\right)$which can originate from $k \geq 2$; and $55 \mathrm{amu}$ $\left(\left(\mathrm{H}_{2} \mathrm{O}\right)_{3} \mathrm{H}^{+}\right)$from $k \geq 4$ (the fragment $\left(\mathrm{H}_{2} \mathrm{O}\right)_{2} \mathrm{H}^{+}$at $37 \mathrm{amu}$ indicative of $k \geq 3$ was obscured by the nearby strong $\mathrm{Ar}^{+}$ peak at $40 \mathrm{amu}$ ). It is interesting to note that here, unlike in the methanol case, the ion fragments containing Ar are observed. Again we measure the pickup pressure dependence of

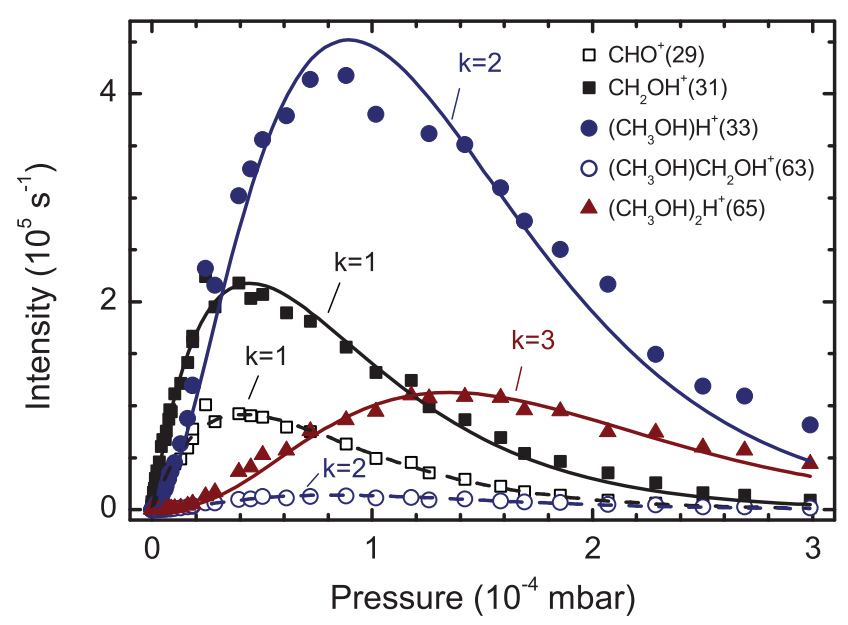

FIG. 3. Intensity of fragment ion peaks in the mass spectra as a function of pickup pressure of $\mathrm{CH}_{3} \mathrm{OH}$. The lines correspond to the Poisson fits $P_{k}$, Eq. (6). Detected ions (mass in amu): $\mathrm{COH}^{+}(29)$ and $\mathrm{CH}_{3} \mathrm{OH}^{+}$(31) fitted with $k=1 ;\left(\mathrm{CH}_{3} \mathrm{OH}\right) \mathrm{H}^{+}(33)$ and $\left(\mathrm{CH}_{3} \mathrm{OH}\right) \mathrm{CH}_{2} \mathrm{OH}^{+}(63)$ fitted with $k=2$; and $\left(\mathrm{CH}_{3} \mathrm{OH}\right)_{2} \mathrm{H}^{+}(65)$ fitted with $k=3$. 


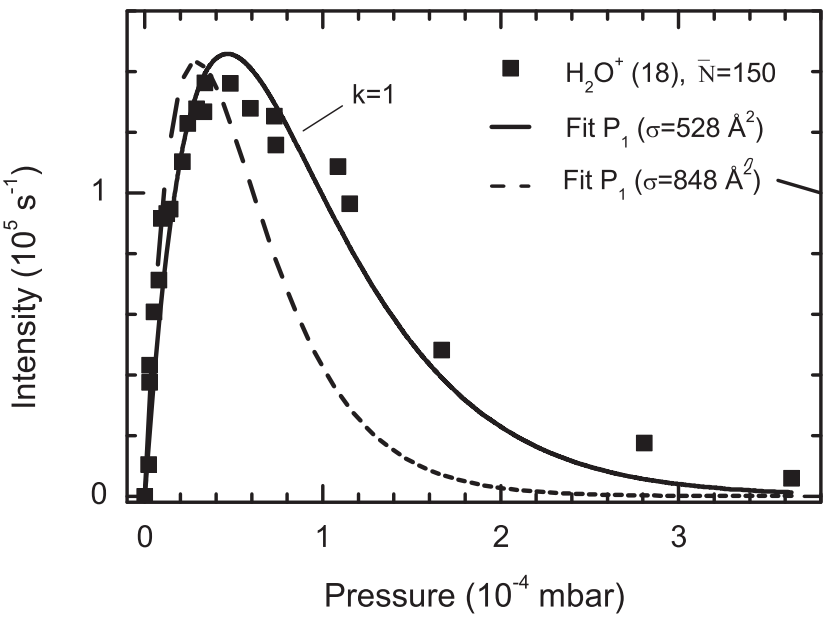

FIG. 4. Intensity of monomer fragment ion peak $(\mathrm{m} / z=18 \mathrm{amu})$ in the mass spectrum as a function of water pressure in the pickup chamber. Solid line represents the Poisson distribution fit (Eq. (6)) with $k=1$ and $\sigma_{e}$ as a fitting parameter $\left(\Rightarrow \sigma_{e}=528 \AA^{2}\right)$. The dashed line corresponds to the Poisson distribution with $\sigma_{e}=848 \AA^{2}$ obtained from the velocity measurement.

$\mathrm{H}_{2} \mathrm{O}^{+}$to monitor $k=1$ pickup and $\left(\mathrm{H}_{2} \mathrm{O}\right) \mathrm{H}^{+}$for $k=2$, and fit these dependencies with the corresponding Poisson statistics.

Finally for $\mathrm{HBr}$ pickup the necessity of a higher mass resolution (to resolve various fragments with ${ }^{79} \mathrm{Br}$ and ${ }^{81} \mathrm{Br}$ isotopes) limited the monitored mass range of our quadrupole below the mass of $150 \mathrm{amu}$. Due to this reason the $\mathrm{HBr}$ cluster ion peaks were not measured. However, an appreciable signal from $\mathrm{H}^{81} \mathrm{BrH}^{+}$at $m / z=83$ amu manifests the presence of $(\mathrm{HBr})_{\mathrm{k}}$ clusters with $k \geq 2$. We have monitored ${ }^{81} \mathrm{Br}$ for the molecule $k=1$ pickup and $\mathrm{H}^{81} \mathrm{BrH}^{+}$peak for $k=2$ capture. The intensity at $81 \mathrm{amu}$ has been corrected for a contribution from $\mathrm{H}^{79} \mathrm{BrH}^{+}$. This could be done since the ${ }^{81} \mathrm{Br}:{ }^{79} \mathrm{Br}$ $=0.973$ ratio is known and the ${ }^{81} \mathrm{HBrH}^{+}$peak was measured.

The experimental data can be illustrated by the example in Fig. 4 which shows the $\mathrm{H}_{2} \mathrm{O}^{+}$ion intensity dependence on the water pickup pressure. The solid line corresponds to the Poisson distribution fit to the data using Eq. (6) with $k=1$ and $\sigma_{e}$ as a fitting parameter. However, the obtained cross section $\sigma_{e} \approx 528 \AA^{2}$ is significantly smaller than $848 \AA^{2}$ obtained for the same clusters from the velocity measurements. If the later value was used for Poisson fit with $k$ $=1$ (dashed line in Fig. 4), the experimental data could not be fitted adequately. Similar results were obtained for the other picked up molecules and the obtained cross sections are summarized in Table I. They will be further discussed in Sec. VI.

Figure 5 shows several examples of pickup data for $\mathrm{HBr}$ (top) and $\mathrm{H}_{2} \mathrm{O}$ (bottom) molecules. Here the data are fitted by a sum of Poisson distributions with constant cross section $\sigma_{e}$ (Eq. (6)) fixed to the value obtained from the velocity measurements. The fitting parameter was the number $m$ of added $P_{k}(p), k=1, \ldots, m$. This is further discussed in Sec. VI.

\section{MOLECULAR DYNAMICS AND MONTE CARLO SIMULATIONS}

In order to support the experimental data we have performed molecular dynamics (MD) and Monte Carlo (MC)

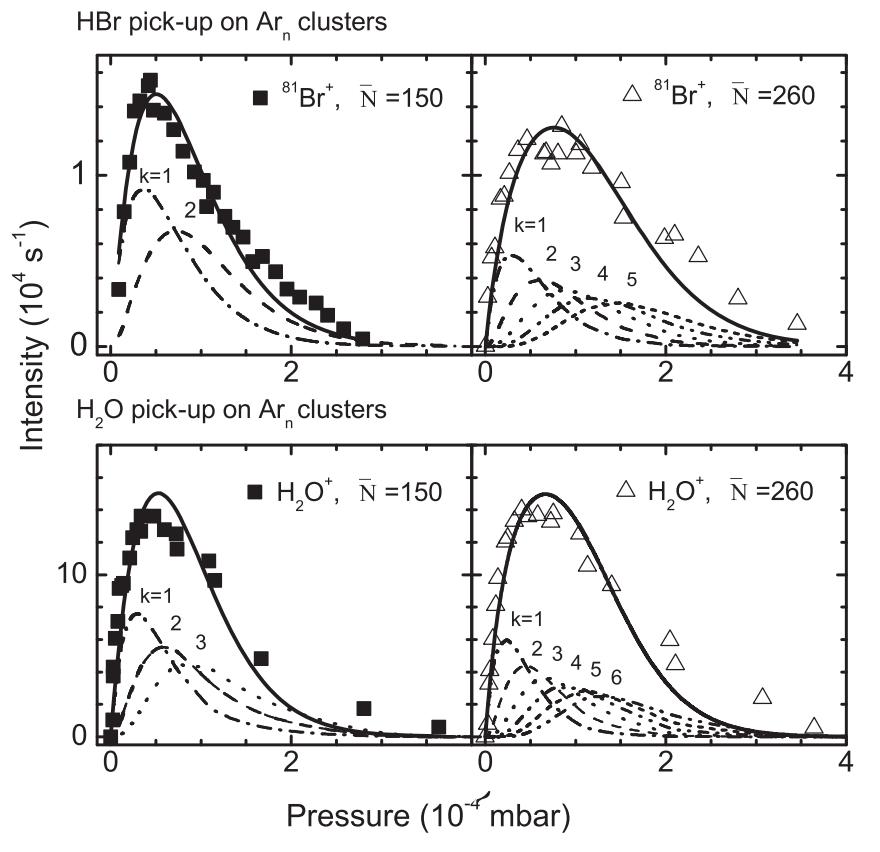

FIG. 5. Intensity of monomer fragment ion peaks in the mass spectra as a function of the pickup chamber pressure. The detected ions were $\mathrm{Br}^{+}(\mathrm{m} / \mathrm{z}$ $=81 \mathrm{amu})$ and $\mathrm{H}_{2} \mathrm{O}^{+}(\mathrm{m} / z=18 \mathrm{amu})$ for $\mathrm{HBr}$ and $\mathrm{H}_{2} \mathrm{O}$ pickup, respectively. The solid lines represent the fits to the data with a sum of Poisson distributions (see Eq. (12)).

simulations of the pickup process. The simulations were performed in the coordinate system in which the cluster is initially at rest and the projectiles were shot at the cluster with the mean velocity corresponding to the experimental cluster beam velocity $\left(490 \mathrm{~ms}^{-1}\right)$. The simulations were performed for the $\operatorname{Ar}_{N}$ cluster size $N=150$ for three projectiles: $\mathrm{Ar}, \mathrm{HBr}$, and $\mathrm{H}_{2} \mathrm{O}$.

The Lennard-Jones (LJ) potential of the form

$$
V_{L J}(r)=4 \varepsilon\left(\left(\frac{\rho}{r}\right)^{12}-\left(\frac{\rho}{r}\right)^{6}\right)
$$

has been used for the description of Ar-Ar, $\mathrm{HBr}-\mathrm{Ar}$, and $\mathrm{H}_{2} \mathrm{O}$ Ar interactions. The parameters $\varepsilon$ and $\rho$ of the potential are listed in Table II. The parameters for Ar-Ar interaction have been obtained in Ref. 49 by fitting the correct pairwise potential of Aziz and Slaman. ${ }^{56}$ It has been shown, that the LJ parameters usually used for simulation of liquids and solids cannot be used for simulations of cryogenic clusters. ${ }^{49}$ Both $\mathrm{HBr}$ and $\mathrm{H}_{2} \mathrm{O}$ were represented as united atom. The parameters for $\mathrm{HBr}-\mathrm{Ar}$ potential were obtained in Ref. 48 by averaging the three body potential of Hutson ${ }^{57}$ over all possible $\mathrm{HBr}$ orientations. The isotropic potential for $\mathrm{H}_{2} \mathrm{O}$-Ar has been successfully used to model argon-water ice collisions. ${ }^{58,59}$ The parameters for $\mathrm{H}_{2} \mathrm{O}-\mathrm{Ar}$ potential were taken from Ref. 58 . The use of isotropic potential is a relatively crude approximation; however, it has been applied previously for MD simulation of pickup of hydrogen halides on rare gas clusters and yielded good agreement with experiments. ${ }^{47,48}$ As seen below, the present simulations agree very well with the experiment also for the $\mathrm{H}_{2} \mathrm{O}-\mathrm{Ar}_{N}$ system, which justifies the use of the isotropic potential also in this case. Because of the relative complexity of the $\mathrm{CH}_{3} \mathrm{OH}$ molecule, the simulation of the 
TABLE II. Lennard-Jones potential parameters used for molecular dynamics simulations.

\begin{tabular}{lcr}
\hline \hline & $\rho$ & $\varepsilon$ \\
& $(\AA)$ & $(\mathrm{K})$ \\
\hline $\mathrm{Ar}-\mathrm{Ar}$ & 3.35 & 143.2 \\
$\mathrm{HBr}-\mathrm{Ar}$ & 3.55 & 174 \\
$\mathrm{H}_{2} \mathrm{O}-\mathrm{Ar}$ & 3.0 & 174 \\
\hline \hline
\end{tabular}

methanol- $\mathrm{Ar}_{N}$ collisions is beyond the scope of the present mostly experimental paper.

We have used the Verlet's algorithm with a time step of $1 \mathrm{fs}$ to integrate Newton's equation of motion. Simulation of one trajectory went as follows: The icosahedral structure of $\mathrm{Ar}_{150}$ cluster was assumed with coordinates taken from Cambridge cluster database. ${ }^{60}$ The temperature of the cluster was assumed to be $32 \mathrm{~K}$ (Ref. 29) and the cluster was equilibrated for $10 \mathrm{ps}$. Then the cluster was randomly oriented and the projectile was shot at the cluster with a certain impact parameter. The impact velocity was sampled from the MaxwellBoltzmann distribution of projectile gas at $300 \mathrm{~K}$ centered around $490 \mathrm{~ms}^{-1}$. For each trajectory, the simulation lasted for $1 \mathrm{~ns}$. After the simulation was finished, it was determined whether the projectile remained on the cluster, how many argon atoms were evaporated from the cluster, and the velocity of the cluster after the collision.

In order to calculate the pickup cross section, 2500 projectile trajectories were generated with random impact parameters and the cross section was evaluated from the ratio of sticking to nonsticking trajectories. This approach is illustrated in Fig. 6. The normally constant maximal impact parameter at which the collision is sticking has been diffused by the fact, that the projectiles have also transversal velocities sampled from the Maxwell-Boltzmann distribution. The pickup cross sections obtained from these simulations are listed in Table I.

In addition to the pickup cross section, the simulations addressed two questions that justify evaluation of the experimental data according to Eq. (5): (i) Are collisions with the gas in the pickup cell elastic or inelastic? (ii) Does the collision lead to an effective evaporation of cluster constituents? The bottom panel of Fig. 6 shows the histogram of simulated final cluster velocities for sticking and nonsticking collisions. The plot demonstrates that a considerable momentum transfer between the projectile and the cluster occurs only in the case of sticking collisions, i.e., in inelastic events. Also, the mean value of the final cluster beam velocity distribution is in excellent agreement with Eq. (3). No evaporation from the cluster was observed on the timescale of the simulation (1 ns).

We have addressed one more question using the simulations: If two $\mathrm{HBr}$ molecules are picked up on the $\mathrm{Ar}_{150}$ cluster, what is the probability that they coagulate, i.e., form the $\mathrm{HBr}$ dimer? The simulation was done as follows: the first $\mathrm{HBr}$ projectile was shot at the cluster with a random impact parameter (within the sticking range) and the system was simulated for 500 ps. Then the second $\mathrm{HBr}$ projectile was shot at this cluster with a random impact parameter and the system was simulated for $1 \mathrm{~ns}$. The distance between the two $\mathrm{HBr}$ atoms
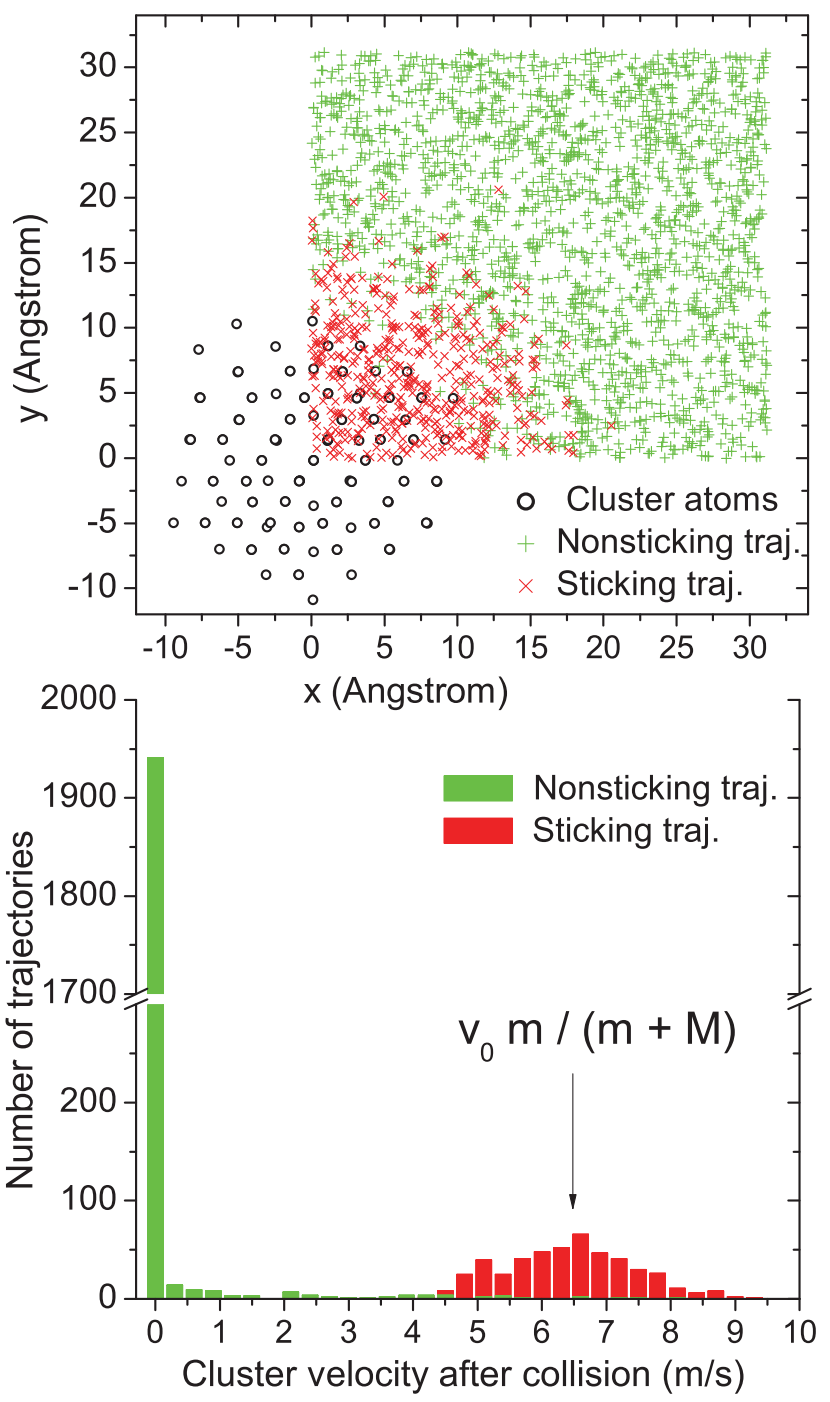

FIG. 6. Results of MD simulations for $\mathrm{Ar}_{150}-\mathrm{HBr}$ collisions. Top: Map of sticking and nonsticking trajectories used to evaluate effective cross section. Bottom: Histogram for cluster velocities after the collisions. In the simulations the $\mathrm{HBr}$ projectiles were shot at the cluster initially in rest with the velocity corresponding to the experimental velocity of the cluster beam. The vertical arrow indicates the velocity shift according to Eq. (3) in excellent agreement with the simulations.

was monitored and if the average distance was less than $5 \AA$ the coagulation was considered to occur (in all cases when the dimer was formed it did not dissociate till the end of the simulation). Total 2500 simulations were run and the coagulation occurred in $22.6 \%$ of cases. The mean time of dimer formation was 93 ps after the impact of the second projectile on the cluster.

The $\mathrm{HBr}-\mathrm{HBr}$ interaction has been approximated by the isotropic hard-core Lennard-Jones (HCLJ) potential of Hurly, ${ }^{61}$

$$
V_{H C L J}(r)=4 \varepsilon\left(\left(\frac{\rho-2 a}{r-2 a}\right)^{12}-\left(\frac{\rho-2 a}{r-2 a}\right)^{6}\right)
$$

with the parameters $\varepsilon=533.45 \mathrm{~K}, \rho=3.44 \AA$, and $a$ $=0.526 \AA$. This potential approximates the $\mathrm{HBr}$ dimer by a diatomic-like molecule with isotropic interaction. We are 
aware of the crudeness of such approximation - the present results do not properly describe the behaviour of two $\mathrm{HBr}$ molecules on Ar cluster. A proper interpretation of the simulation is that it describes the behaviour of two picked up atom-like objects that have the same binding energy as the $\mathrm{HBr}$ dimer. Nonetheless, results of such simulation - coagulation only in about $23 \%$ of cases - are helpful to understand the discrepancy in the experimental methods (see the discussion in Sec. VI A).

\section{DISCUSSION}

\section{A. Capture cross sections}

The major point to be discussed here is the comparison of the pickup cross sections summarized in Table I. First, we focus on the effective cross sections $\sigma_{e}$ evaluated from the velocity measurements which are in a good agreement with the simulated ones: in all cases the calculated cross sections exceed the measured ones by about $10 \%$ for $\mathrm{HBr}$ and $\mathrm{H}_{2} \mathrm{O}$ and by $14 \%$ for Ar. The agreement between the velocity measurements and simulations suggests that the cross sections determined by this method are close to the real values.

It is interesting to note that $\sigma_{0}$ is consistent for all the molecules except methanol; i.e., $\sigma_{0} \approx 570 \AA^{2}$ for $\bar{N}=150$ with all the values within $4 \%$, and $\sigma_{0} \approx 775 \AA^{2}$ for $\bar{N}$ $=260$ with all the values within $10 \%$. For methanol significantly larger $\sigma_{0}$ were obtained which could be caused by the fact that velocity correction factors corresponding to the hard sphere potential approximation is not valid for this large molecule with internal structure and many degrees of freedom. It should be also noted that the simulated cross sections were calculated in a way that mimics the experiment, thus they correspond to the effective cross section $\sigma_{e}$, i.e., the velocity correction factors $F_{a 0}$ are implicitly included. Both, in the experiment and simulations the cross sections are obtained on the assumption, that the projectile is a point-like particle, i.e., the size of the incoming molecule is neglected, or more precisely included in the obtained $\sigma_{e}$. This may add a small relative shift, especially for molecules like methanol.

We also compare the obtained cross sections to the simple geometrical cross sections. To determine the cluster mean size $\bar{N}$ by any of the two methods exploited here a certain analytical relationship between the cross section $\sigma$ and $N$ had to be assumed. Both Cuvellier et al. ${ }^{36}$ and Macler and Bae, ${ }^{3}$ assumed a geometrical pickup cross sections when introducing their methods for the mean cluster size determination, i.e.,

$$
\sigma_{g}=\pi R_{N}^{2}, \quad R_{N}=\left(\frac{3 a^{3}}{16 \pi} N\right)^{1 / 3},
$$

where $a=5.34 \AA$ is the lattice parameter of Ar clusters. ${ }^{29}$ The geometrical cross sections corresponding to the present mean cluster sizes $\bar{N}=150$ and 260 are $\sigma_{g}=390 \AA^{2}$ and $560 \AA^{2}$, respectively. Cuvellier et al. ${ }^{36}$ used collision dynamics simulations to account for a more realistic attractive interaction of the molecule with the cluster and modified the geometrical cross section formula to

$$
\sigma_{g 0}=\pi\left(R_{N}+R_{0}\right)^{2} .
$$

The value $R_{0}=3 \AA$ was determined by the simulations of Ar$\operatorname{Ar}_{125}$ system. This yields the geometrical cross section $\sigma_{g 0}$ $=620 \AA^{2}$ and $840 \AA^{2}$ for the present mean cluster sizes $\bar{N}$ $=150$ and 260 , respectively.

Clearly the simple hard sphere cross sections $\sigma_{g}$ (Eq. (10)) are strongly underestimated. Adopting the approach of Cuvellier et al. ${ }^{36}$ we can modify $\sigma_{g}$ by the contribution $R_{0}$ due to the interaction between the cluster and the projectile to $\sigma_{g 0}$ (Eq. (11)). Thus from comparison of the simulated cross sections with $\sigma_{g}$, the parameter $R_{0}$ can be evaluated (Table I). Note, that the comparison was made with the calculated $\sigma_{e}$ value which implicitly includes also the velocity dependence $\left(F_{a 0}\right)$ thus $R_{0}$ is in principle velocity dependent. Since this parameter is due to the interaction between the species, it should correlate with the potential parameters in Table II. It is interesting to note that $R_{0}$ increases with the decreasing parameter $\rho$ of LJ potential.

The most striking observation is the significantly smaller cross sections evaluated from the Poisson distributions. The Poisson statistics is frequently used to determine the number $k$ of particles picked up by the cluster. ${ }^{2,7,16,19}$ Generally the good quality of the Poisson distribution fit to the experimental data is regarded as the justification for using such analysis to obtain $k, \bar{N}$, or $\sigma$ values. The present measurements show that, although high quality fits could be obtained, the evaluated cross sections still differ significantly from the real values. An indication that the $\sigma_{e}$ values are wrong is the fact that they are larger for the smaller clusters in all cases.

Also the second order $k=2$ Poisson distributions were fitted to the corresponding signals for all three studied molecules, e.g., at masses $m / z=19$ amu $\left[\mathrm{H}_{2} \mathrm{OH}^{+}\right]$, $33 \mathrm{amu}\left[\left(\mathrm{CH}_{3} \mathrm{OH}\right) \mathrm{H}^{+}\right]$, and $83 \mathrm{amu}\left[\mathrm{HBr}^{81} \mathrm{H}^{+}\right]$. However, even smaller cross sections were obtained from these fits than from the $k=1$ fragments. The quality of these fits was somewhat worse with evident broadening in the high pressure region.

Below several factors are addressed that complicate the emergence of the Poisson statistics and could explain why the analysis yields the lower cross sections. We argue that some of these factors (i.e., non-sticking collisions, evaporation, and scattering from the beam) do not contribute to present discrepancy, while other factors (i.e., coagulation and fragmentation upon ionization) can contribute.

The first possible reason for the discrepancy could be the non-sticking collisions. If the sticking coefficient of a molecule is $s<1$, only a fraction of the total number of collisions will actually result in the capture of the molecule on the cluster. On the other hand, the cluster can be slowed down even in an inelastic collision, which does not result in complete momentum transfer and the sticking of the molecule to the cluster. However, as is demonstrated in Fig. 6, the simulations suggest that the measured velocity decrease is caused almost exclusively by the sticking collisions, and thus the cross sections obtained by both methods should be the same.

Further effect can be an evaporation of the Ar cluster atoms due to the energy transfer in the sticking collision. This effect would lower the cross section for each subsequent pickup event, since $\sigma \propto \bar{N}^{2 / 3}$. The molecular 
dynamics simulations did not show any evaporation within $1 \mathrm{~ns}$ after the pickup. The large clusters are efficient thermal baths for energy dissipation and a significant evaporation on longer timescale is not expected. The following energy arguments can be also used. For the binding energy of a single Ar atom to the $\operatorname{Ar}_{N}$ cluster $N=150$ a model calculation ${ }^{62}$ as well as our estimate based on the LJ potential yield $\sim 80 \mathrm{meV}$. The binding energy of an $\mathrm{HBr}$ molecule to the cluster can be estimated from the $\operatorname{Ar}-\mathrm{Ar}_{N}$ binding energy and the comparison of the LJ potentials for Ar-Ar and $\mathrm{HBr}-\mathrm{Ar}$ interactions (Table II), i.e., the $\mathrm{HBr}-\mathrm{Ar}_{N}$ energy would correspond to $\sim 100 \mathrm{meV}$. Upon the pickup of a single $\mathrm{HBr}$ molecule this binding energy plus the heat due to collision inelasticity $(\sim 100 \mathrm{meV})$ are transferred to the cluster, i.e., total about $200 \mathrm{meV}$ which would be sufficient to evaporate only two Ar atoms. This effect could not explain the observed cross section decrease.

The scattering of clusters on molecules in the pickup cell can deflect the smaller clusters out of the beam to a greater extent than the large ones, and thus shift the mean cluster size to a larger value. This effect could make the observed capture cross section larger which, however, is not the case. ${ }^{63}$ Additionally, this effect would influence both experimental methods simultaneously.

One explanation of the observed difference concerns the coagulation of the embedded molecules. It was discussed in detail for He clusters, ${ }^{2-4}$ however, the present case of solidlike argon clusters might differ significantly from the superfluid helium nanodroplets. The coagulation on Ar clusters will be controlled by a competition between the polarization forces attracting the molecules towards each other and the forces binding the molecules to the cluster and hindering their diffusion on the surface. This issue has been also addressed with our MD simulations (Sec. V) for two HBr-like objects picked up by the cluster. The coagulation (i.e, the formation of $\mathrm{HBr}$ dimer on the cluster surface) occurred only in $22.6 \%$ out of 2500 simulations. If the picked up molecules do not coagulate, even the pickup of $k \geq 2$ molecules will contribute to the signal assigned to the monomer. This signal is, however, fitted with $k=1$ distribution $P_{k}(p)$. Thus the ion signal dependence corresponding to the monomer will be a composition of several independent events where $k=1,2, \ldots$ molecules are picked up by the cluster. Figure 5 shows the experimental data fitted with a sum of Poisson distributions,

$$
P(p)=A_{0} \sum_{k=1}^{m} P_{k}(p)
$$

In the nonlinear least-squares fit procedure the number $m$ of $P_{k}$ distributions (Eq. (6)) was varied while the parameter $\sigma$ was fixed to the value obtained from the velocity experiment. From these fits it would follow that Ar clusters of size $\bar{N}=150$ can accommodate up to $m=3$ water molecules without coagulation. The distribution for larger clusters is even broader with up to $m=6$ water molecules. This would imply very poor migration of the guest molecule on the Ar cluster.
Yet another important issue is the fragmentation of the host molecule or cluster upon the ionization. The assumption that we measure the Poisson distribution for the pickup of $k$ molecules $\mathrm{X}$ can be only valid if we detect a fragment which corresponds exclusively to the ionization of $\mathrm{X}_{\mathrm{k}}$ species, i.e., for $P_{1}$ distribution we have to choose the fragment which corresponds exclusively to the monomer ionization and not to the ionization of an $\mathrm{X}_{k \geq 2}$ cluster. However, the fragmentation dynamics can be more complicated and affected by the $\mathrm{Ar}$ cluster. For example, the accepted fast proton transfer reaction as a main fragmentation channel for the hydrogen bonded clusters after ionization can be quenched by the cluster environment as observed for the methanol clusters on larger Ar clusters. ${ }^{18}$ Thus, even if the molecules coagulate and form the dimer, upon ionization the dimer fragment can contribute to $P_{1}$ distribution and the fit will yield erroneous result for the cross section.

In summary, due to incomplete coagulation and ionization fragmentation dynamics the pickup of $k \geq 2$ molecules can still contribute to the measured intensity of the fragment assigned to the pickup of a single molecule. The dependence of the fragment intensity on the pickup pressure will thus extend to higher pressures which will result in smaller fitted $\sigma_{e}$ if the fit with a single Poisson $(k=1)$ distribution is used. It ought to be mentioned that in some cases dependencies with clearly enhanced intensity at higher pressures have been measured. Thus the pickup cross section evaluated from the Poisson distribution fit will be smaller than the real cross section.

\section{B. Implications for cluster size determination}

Two methods for the mean cluster size determination based on the pickup process were examined in our study. ${ }^{36,37}$ Since the cluster mean size $\bar{N}$ and the pickup cross section $\sigma$ cannot be determined independently by these methods, both methods assumed a certain analytical relationship between these two quantities which was the geometrical cross section of a hard sphere. Our original motivation for these measurements was that by combining Eqs. (5) and (6), both quantities $\sigma$ and $\bar{N}$ could be determined by simultaneous measurements of velocity and Poisson distributions. This would present a new method for determining the mean cluster size in a beam without the geometrical cross section assumption.

However, as seen above, in the course of our pickup measurements we have discovered that the two methods actually provide inconsistent results. The reason is that in the Poisson distribution measurements, incomplete coagulation of molecules on the cluster surface and their fragmentation patterns complicate the fitting procedure. Since these processes are not always obvious, it has to be cautiously examined which species are actually detected in the Poisson distribution measurements, if the cluster size or cross section are to be determined from such data. In other words, if the mean cluster size or cluster cross section is based solely on the Poisson distribution $P_{k}(p)$ measurement of a certain fragment after the pickup process, the result can be wrong, unless the measured fragment ion is assigned unambiguously to the 
pickup of $k$ molecules and any contribution from pickup of more than $k$ molecules is excluded.

\section{CONCLUSIONS}

We report here the capture cross sections $\sigma_{e}$ for pickup of several gas molecules $\left(\mathrm{HBr}, \mathrm{H}_{2} \mathrm{O}, \mathrm{CH}_{3} \mathrm{OH}\right)$ and $\mathrm{Ar}$ atoms on $\operatorname{Ar}_{N}$ clusters of the mean sizes $\bar{N} \approx 150$ and 260. Two different methods proposed in the literature ${ }^{36,37}$ were employed in the present measurements and $\sigma_{e}$ was evaluated from

1. the decrease in the cluster beam velocity due to the momentum transfer of the picked up molecules;

2. the Poisson distribution fit of a selected cluster fragment ion intensity dependence on the pickup pressure.

In addition, the pickup cross sections were calculated with molecular dynamics and Monte Carlo simulations. The simulations support the results of the velocity measurements. On the other hand, the Poisson distributions yield significantly smaller cross sections, which are also inconsistent with the dependence on the cluster size. The reasons for the inconsistency of the results based on the Poisson distributions are discussed in terms of (i) an incomplete coagulation of guest molecules on the argon clusters when $k \geq 2$ molecules are picked up, and (ii) the fragmentation pattern of the embedded molecules and their clusters upon ionization on the Ar cluster.

The general message from this paper is that the Poisson distribution method has to be cautiously examined, if conclusions should be drawn about the cluster mean size $\bar{N}$, or the cross section $\sigma$, or the number $k$ of picked up molecules.

It is also important to note that both the velocity measurements as well as the MC simulations show that the simple geometrical cross sections are significantly smaller than the real values and therefore not a good approximation for the cluster pickup cross section.

\section{ACKNOWLEDGMENTS}

This work has been supported by the Grant Agency of the Czech Republic Project Nos. 203/09/0422 and P208/11/0161, and the Academy of Sciences of the Czech Republic Project No. KJB400400902. J. Fedor acknowledges the support of the Grant No. 235414 "IPhoN" within FP7-MC-IEF. We gratefully acknowledge discussing our results with Udo Buck.

${ }^{1}$ T. E. Gough, M. Mengel, P. A. Rowntree, and G. Scoles, J. Chem. Phys. 83, 4958 (1985).

${ }^{2}$ M. Lewerenz, B. Schilling, and J. P. Toennies, J. Chem. Phys. 102, 8191 (1995).

${ }^{3}$ M. Macler and Y. K. Bae, J. Chem. Phys. 106, 5785 (1997).

${ }^{4}$ M. Lewerenz, B. Schilling, and J. P. Toennies, J. Chem. Phys. 106, 5787 (1997).

${ }^{5}$ M. Hartmann, R. E. Miller, J. P. Toennies, and A. F. Vilesov, Phys. Rev. Lett. 75, 1566 (1995).

${ }^{6}$ S. Grebenev, J. P. Toennies, and A. F. Vilesov, Science 279, 2083 (1998).

${ }^{7}$ M. Behrens, R. Fröchtenicht, M. Hartmann, J. G. Siebers, and U. Buck, J. Chem. Phys. 111, 2436 (1999).

${ }^{8}$ G. Scoles and K. K. Lahmann, Science 287, 2429 (2000).

${ }^{9}$ J. P. Toennies and A. F. Vilesov, Angew. Chem., Int. Ed. 43, 2622 (2004).

${ }^{10}$ M. Y. Choi, G. E. Douberly, T. M. Falconer, W. K. Lewis, C. M. Lindsay, J. M. Merritt, P. L. Stiles, and R. E. Miller, Int. Rev. Phys. Chem. 25, 15 (2006).
${ }^{11}$ E. Lugovoj, J. P. Toennies, and A. F. Vilesov, J. Chem. Phys. 112, 8217 (2000).

${ }^{12}$ M. Fárník and J. P. Toennies, J. Chem. Phys. 122, 014307 (2005).

${ }^{13}$ J. M. Merritt, S. Rudić, and R. E. Miller, J. Chem. Phys. 124, 084301 (2006).

${ }^{14}$ S. Denifl, F. Zappa, I. Mähr, F. Ferreira da Silva, A. Aleem, A. Mauracher, M. Probst, J. Urban, P. Mach, A. Bacher, O. Echt, T. D. Märk, and P. Scheier, Angew. Chem., Int. Ed. 121, 9102 (2009).

${ }^{15}$ A. Gutberlet, G. Schwaab, O. Birer, M. Masia, A. Kaczmarek, H. Forbert, M. Havenith, and D. Marx, Science 324, 1545 (2009).

${ }^{16}$ J. M. Mestdagh, M. A. Gaveau, C. Gée, O. Sublemontier, and J. P. Visticot, Int. Rev. Phys. Chem. 16, 215 (1997).

${ }^{17}$ M.-A. Gaveau, E. Glogauen, P.-R. Fournier, and J.-M. Mestdagh, J. Phys. Chem. A 109, 9494 (2005).

${ }^{18}$ M. Ehbrecht, M. Stemmler, and F. Huisken, Int. J. Mass. Spectrom. 123, R1 (1993).

${ }^{19}$ U. Buck, J. Phys. Chem. A 106, 10049 (2002).

${ }^{20}$ V. Poterya, M. Fárník, P. Slavíček, U. Buck, and V. V. Kresin, J. Chem. Phys. 126, 071101 (2007).

${ }^{21}$ V. Poterya, O. Votava, M. Fárník, M. Ončák, P. Slavíček, U. Buck, and B. Friedrich, J. Chem. Phys. 128, 104313 (2008).

${ }^{22}$ V. Poterya, J. Fedor, A. Pysanenko, O. Tkáč, J. Lengyel, M. Ončák, P. Slavíček, and M. Fárník, Phys. Chem. Chem. Phys. 13, 2250 (2011).

${ }^{23}$ C. Nitsch, C. P. Schulz, A. Gerber, W. Zimmermann-Edling, and I. V. Hertel, Z. Phys. D: At., Mol. Clusters 22, 651 (1992).

${ }^{24}$ M. Ahmed, C. J. Apps, C. Hughes, N. E. Watt, and J. C. Whitehead, J. Phys. Chem. A 101, 1250 (1997).

${ }^{25} \mathrm{H}$. Haberland, Clusters of Atoms and Molecules (Springer, Berlin, 1994).

${ }^{26}$ U. Buck and H. Meyer, Phys. Rev. Lett. 52, 109 (1984).

${ }^{27}$ U. Buck and H. Meyer, J. Chem. Phys. 84, 4854 (1986).

${ }^{28}$ T. D. Märk, in Linking the Gaseous and Condensed Phases of Matter, edited by L. G. Christopherou (Plenum, New York, 1994), p. 155.

${ }^{29}$ J. Farges, M. F. de Feraudy, B. Raoult, and G. Torchet, Sur. Sci. 106, 95 (1981).

${ }^{30}$ J. Farges, M. F. de Feraudy, B. Raoult, and G. Torchet, J. Chem. Phys. 78, 5067 (1983).

${ }^{31}$ G. Torchet, P. Schwartz, J. Farges, M. F. de Feraudy, and B. Raoult, J. Chem. Phys. 79, 6196 (1983).

${ }^{32}$ J. Farges, M. F. de Feraudy, B. Raoult, and G. Torchet, J. Chem. Phys. 84, 3491 (1986)

${ }^{33}$ A. D. Martino, M. Benslimane, M. Châtelet, C. Crozes, F. Padère, and H. Vach, Z. Phys. D: At., Mol. Clusters 27, 185 (1993).

${ }^{34}$ C. Bobbert, S. Schütte, C. Steinbach, and U. Buck, Eur. Phys. J. D 19, 183 (2002).

${ }^{35}$ U. Buck and R. Krohne, J. Chem. Phys. 105, 5408 (1996).

${ }^{36}$ J. Cuvellier, P. Meynadier, P. de Pujo, O. Sublemontier, J.-P. Visticot, J. Berlande, A. Lallement, and J.-M. Mestdagh, Z. Phys. D: At., Mol. Clusters 21, 265 (1991).

${ }^{37}$ M. Macler and Y. K. Bae, J. Phys. Chem. A 101, 145 (1997).

${ }^{38}$ O. F. Hagena, Surf. Sci. 106, 101 (1981).

${ }^{39}$ O. F. Hagena, Z. Phys. D: At., Mol. Clusters 4, 291 (1987).

${ }^{40}$ O. F. Hagena, Rev. Sci. Instrum. 63, 2374 (1992).

${ }^{41}$ R. Baumfalk, N. H. Nahler, U. Buck, M. Y. Niv, and R. B. Gerber, J. Chem. Phys. 113, 329 (2000).

${ }^{42}$ R. Baumfalk, U. Buck, C. Frischkorn, S. R. Gandhi, and C. Lauenstein, Ber. Bunsenges. Phys. Chem. 101, 606 (1997).

${ }^{43}$ M. Fárník, Molecular Dynamics in Free Clusters and Nanoparticles Studied in Molecular Beams (ICT Prague Press, Institute of Chemical Technology Prague, 2011).

${ }^{44}$ The experiments were also performed with a small pickup cell of $L=5$ $\mathrm{cm}$ length placed in the next vacuum chamber. Since the capture probability is proportional to $L$ and the pickup gas pressure $p$, using the chamber with longer pickup path allowed to lower the pressure $p$ resulting in a more precise pressure control and consequently better characterization of the pickup process. The results using the two different chambers were consistent within the experimental error.

${ }^{45}$ D. J. Auerbach, in Atomic and Molecular Beam Methods, edited by G. Scoles (Oxford University Press, New York, 1988), Vol. I, p. 362.

${ }^{46}$ H. Pauly, Atom, Molecule and Cluster Beams (Springer, Berlin, 2000).

${ }^{47}$ M. Fárník, N. H. Nahler, U. Buck, P. Slavíček, and P. Jungwirth, Chem. Phys. 315, 161 (2005).

${ }^{48}$ N. H. Nahler, R. Baumfalk, U. Buck, H. Vach, P. Slavíček, and P. Jungwirth, Phys. Chem. Chem. Phys. 5, 3394 (2003). 
${ }^{49}$ P. Slavíček, P. Jungwirth, M. Lewerenz, N. H. Nahler, M. Fárník, and U. Buck, J. Chem. Phys. 120, 4498 (2004).

${ }^{50}$ K. Berling, R. Belbing, K. Kramer, H. Pauly, C. Schlier, and P. Toschek, Z. Phys. 166, 406 (1962).

${ }^{51}$ N. C. Lang, H. V. Lilenfeld, and J. L. Kinsey, J. Chem. Phys. 55, 3114 (1971).

${ }^{52}$ U. Nagashima, H. Shinohara, N. Nishi, and H. Tanaka, J. Chem. Phys. 84 209 (1986)

${ }^{53}$ S. Morgan and A. W. Castleman, J. Phys. Chem. 93, 4544 (1989).

${ }^{54}$ F. Huisken and M. Stemmler, Z. Phys. D: At., Mol. Clusters 24, 277 (1992).

${ }^{55}$ U. Buck and M. Winter, Z. Phys. D: At., Mol. Clusters 31, 291 (1994).

${ }^{56}$ R. A. Aziz and M. J. Slaman, Mol. Phys. 58, 679 (1986).

${ }^{57}$ J. M. Hutson, J. Chem. Phys. 91, 4455 (1989).

${ }^{58}$ K. Bolton, M. Svanberg, and J. B. C. Pettersson, J. Chem. Phys. 110, 5380 (1999).
${ }^{59}$ P. U. Andersson, M. B. Någård, K. Bolton, M. Svanberg, and J. B. C. Pettersson, J. Phys. Chem. A 104, 2681 (2000).

${ }^{60}$ D. J. Wales, J. P.K. Doye, A. Dullweber, M. P. Hodges, F. Y. Naumkin, F. Calvo, J. Hernández-Rojas, and T. F. Middleton, The Cambridge Cluster Database, available from http://www-wales.ch.cam.ac.uk/CCD.html.

${ }^{61}$ J. J. Hurly, Int. J. Thermophys. 21, 805 (2000).

${ }^{62}$ K. Hansen and E. E.B. Cambell, Int. J. Mass. Spectrom. 233, 215 (2004).

${ }^{63} \mathrm{We}$ attempted to correct the data for the beam attenuation by using an exponential fit to observed decrease of the total pressure in the detector chamber. This results in somewhat larger evaluated cross sections, yet still not in agreement with the velocity measurements. Since only the small clusters can be deflected enough to escape the detection, the estimated error of the obtained cross sections due to this effect could be $\lesssim 10 \%$. 